\title{
Improving the Price of Anarchy for Selfish Routing via Coordination Mechanisms
}

\author{
George Christodoulou* $^{*} \quad$ Kurt Mehlhorn $^{\dagger} \quad$ Evangelia Pyrga $^{\ddagger}$
}

\begin{abstract}
We reconsider the well-studied Selfish Routing game with affine latency functions. The Price of Anarchy for this class of games takes maximum value $4 / 3$; this maximum is attained already for a simple network of two parallel links, known as Pigou's network. We improve upon the value 4/3 by means of Coordination Mechanisms.

We increase the latency functions of the edges in the network, i.e., if $\ell_{e}(x)$ is the latency function of an edge $e$, we replace it by $\hat{\ell}_{e}(x)$ with $\ell_{e}(x) \leq \hat{\ell}_{e}(x)$ for all $x$. Then an adversary fixes a demand rate as input. The engineered Price of Anarchy of the mechanism is defined as the worst-case ratio of the Nash social cost in the modified network over the optimal social cost in the original network. Formally, if $\hat{C}_{N}(r)$ denotes the cost of the worst Nash flow in the modified network for rate $r$ and $C_{\text {opt }}(r)$ denotes the cost of the optimal flow in the original network for the same rate then

$$
e P o A=\max _{r \geq 0} \frac{\hat{C}_{N}(r)}{C_{\text {opt }}(r)} .
$$

We first exhibit a simple coordination mechanism that achieves for any network of parallel links an engineered Price of Anarchy strictly less than 4/3. For the case of two parallel links our basic mechanism gives $5 / 4=1.25$. Then, for the case of two parallel links, we describe an optimal mechanism; its engineered Price of Anarchy lies between 1.191 and 1.192.
\end{abstract}

\section{Introduction}

We reconsider the well-studied Selfish Routing game with affine cost functions and ask whether increasing the cost functions can reduce the cost of a Nash flow. In other words, the increased cost functions should induce a user behavior that reduces cost despite the fact that the cost is now determined by increased cost functions. We answer the question positively in the following sense. The Price of Anarchy, defined as the maximum ratio of Nash cost to optimal cost, is $4 / 3$ for this class of games. We show that increasing costs can reduce the price of anarchy to a value strictly below $4 / 3$ at least for the case of networks of parallel links. For a network of two parallel links, we reduce the price of anarchy to a value between 1.191 and 1.192 and prove that this is optimal. In order to state our results precisely, we need some definitions.

We consider single-commodity congestion games on networks, defined by a directed graph $G=$ $(V, E)$, designated nodes $s, t \in V$, and a set $\ell=\left(\ell_{e}\right)_{e \in E}$ of non-decreasing, non-negative functions; $\ell_{e}$ is the latency function of edge $e \in E$. Let $P$ be the set of all paths from $s$ to $t$, and let $f(r)$ be a feasible $s, t$-flow routing $r$ units of flow. For any $p \in P$, let $f_{p}(r)$ denote the amount of flow that $f(r)$ routes via path $p$. For ease of notation, when $r$ is fixed and clear from context, we will write simply $f, f_{p}$ instead of $f(r), f_{p}(r)$. By definition, $\sum_{p \in P} f_{p}=r$. Similarly, for any edge $e \in E$, let $f_{e}$ be the amount of flow

\footnotetext{
${ }^{*}$ University of Liverpool, United Kingdom. gchristo@liv. ac.uk

${ }^{\dagger}$ Max-Planck-Institut für Informatik, Saarbrücken, Germany. mehlhorn@mpi-inf .mpg. de

†Technische Universität München, Germany. pyrga@in.tum.de
} 


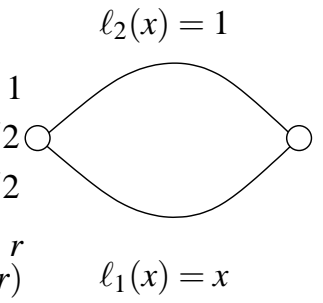

(a)

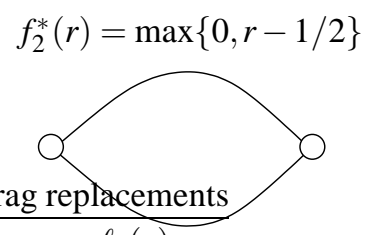

$f_{1}^{*}(r)=\min \{1 / 2, r\}$

(b)

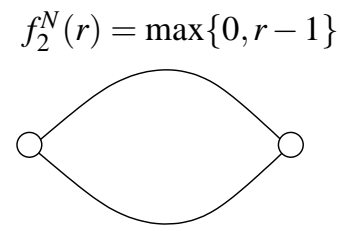

$f_{1}^{N}(r)=\min \{1, r\}$

(c)

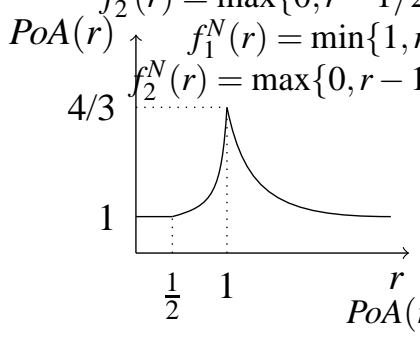

(d)

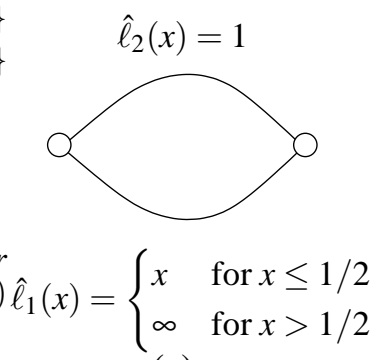

(e)

Figure 1: Pigou's network: We show the original network in (a), the optimal flow in (b) and the Nash flow in (c) as a function of the rate $r$, respectively. The Price of Anarchy as a function of the rate is shown in (d); $\operatorname{PoA}(r)$ is 1 for $r \leq 1 / 2$, then starts to grow until it reaches its maximum of $4 / 3$ at $r=1$, and then decreases again and approaches 1 as $r$ goes to infinity. Finally, in (e) we show the modified latency functions. We obtain $e P o A(r)=1$ for all $r$ in the case of Pigou's network.

going through $e$. We define the latency of $p$ under flow $f$ as $\ell_{p}(f)=\sum_{e \in p} \ell_{e}\left(f_{e}\right)$ and the cost of flow $f$ as $C(f)=\sum_{e \in E} f_{e} \cdot \ell_{e}\left(f_{e}\right)$ and use $C_{\text {opt }}(r)$ to denote the minimum cost of any flow of rate $r$. We will refer to such a minimum cost flow as an optimal flow (Opt). A feasible flow $f$ that routes $r$ units of flow from $s$ to $t$ is at Nash (or Wardrop [29]) Equilibrium 1 if for $p_{1}, p_{2} \in P$ with $f_{p_{1}}>0, \ell_{p_{1}}(f) \leq \ell_{p_{2}}(f)$. We use $C_{N}(r)$ to denote the maximum cost of a Nash flow for rate $r$. The Price of Anarchy (PoA) [23] (for demand $r$ ) is defined as

$$
\operatorname{PoA}(r)=\frac{C_{N}(r)}{C_{o p t}(r)} \quad \text { and } \quad P o A=\max _{r>0} \operatorname{PoA}(r)
$$

PoA is bounded by $4 / 3$ in the case of affine latency functions $\ell_{e}(x)=a_{e} x+b_{e}$ with $a_{e} \geq 0$ and $b_{e} \geq 0$; see [28, 13]. The worst-case is already assumed for a simple network of two parallel links, known as Pigou's network; see Figure 1 .

A Coordination Mechanism 2 replaces the cost functions $\left(\ell_{e}\right)_{e \in E}$ by functions $3 \hat{\ell}=\left(\hat{\ell}_{e}\right)_{e \in E}$ such that $\hat{\ell}_{e}(x) \geq \ell_{e}(x)$ for all $x \geq 0$. Let $\hat{C}(f)$ be the cost of flow $f$ when for each edge $e \in E$, $\hat{\ell}_{e}$ is used instead of $\ell_{e}$ and let $\hat{C}_{N}(r)$ be the maximum cost of a Nash flow of rate $r$ for the modified latency functions. We define the engineered Price of Anarchy (for demand $r$ ) as

$$
e P o A(r)=\frac{\hat{C}_{N}(r)}{C_{o p t}(r)} \quad \text { and } \quad e P o A=\max _{r>0} e P o A(r) .
$$

\footnotetext{
${ }^{1}$ This assumes continuity and monotonicity of the latency functions. For non-continuous functions, see the discussion later in this section.

${ }^{2}$ Technically, we consider symmetric coordination mechanisms in this work, as defined in [9] i.e., the latency modifications affect the users in a symmetric fashion.

${ }^{3}$ One can interpret the difference $\hat{\ell}_{e}-\ell_{e}$ as a flow-dependent toll imposed on the edge $e$.
} 
We stress that the optimal cost refers to the original latency functions $\ell$.

Non-continuous Latency Functions: In the previous definition, as it will become clear in Section 2 , it is important to allow non-continuous modified latencies. However, when we move from continuous to non-continuous latency functions, Wardrop equilibria do not always exist. Non-continuous functions have been studied by transport economists to model the effects of step-function congestion tolls and traffic lights. Several notions of equilibrium that handle discontinuities have been proposed in the literature 4 . The ones that are closer in spirit to Nash equilibria, are those proposed by Dafermos [15] and Berstein and Smith [4]. According to the Dafermos' [15] definition of user optimization, a flow is in equilibrium if no sufficiently small fraction of the users on any path, can decrease the latency they experience by switching to another path6. Berstein and Smith [4] introduced the concept of User Equilibrium, weakening further the Dafermos equilibrium, taking the fraction of the users to the limit approaching 0 . The main idea of their definition is to capture the notion of the individual commuter, that is implicit in Wardrop's definition for continuous functions. The Dafermos equilibrium on the other hand is a stronger concept that captures the notion of coordinated deviations by groups of commuters.

We adopt the concept of User Equilibrium. Formally, we say that a feasible flow $f$ that routes $r$ units of flow from $s$ to $t$ is a User Equilibrium, iff for all $p_{1}, p_{2} \in P$ with $f_{p_{1}}>0$,

$$
\ell_{p_{1}}(f) \leq \liminf _{\varepsilon \downarrow 0} \ell_{p_{2}}\left(f+\varepsilon \mathbf{1}_{p_{2}}-\varepsilon \mathbf{1}_{p_{1}}\right),
$$

where $\mathbf{1}_{p}$ denotes the flow where only one unit passes along a path $p$.

Note that for continuous functions the above definition is identical to the Wardrop Equilibrium. One has to be careful when designing a Coordination Mechanism with discontinuous functions, because the existence of equilibria is not always guaranteed7. It is important to emphasize, that all the mechanisms that we suggest in this paper use both lower semicontinuous and regulat 8 latencies, and therefore User Equilibrium existence is guaranteed due to the theorem of [4]. Moreover, since our modified latencies are non-decreasing, all User Equilibria are also Dafermos-Sparrow equilibria. From now on, we refer to the User Equilibria as Nash Equilibria, or simply Nash flows.

Our Contribution: We demonstrate the possibility of reducing the Price of Anarchy for Selfish Routing via Coordination Mechanisms. We obtain the following results for networks of $k$ parallel links.

- if original and modified latency functions are continuous, no improvement is possible, i.e., $e P o A \geq$ $P o A ;$ see Section 2

- for the case of affine cost functions, we describe a simple coordination mechanism that achieves an engineered Price of Anarchy strictly less than 4/3; see Section 3 The functions $\hat{\ell}_{e}$ are of the form

$$
\hat{\ell}_{e}(x)= \begin{cases}\ell_{e}(x) & \text { for } x \leq r_{e} \\ \infty & \text { for } x>r_{e}\end{cases}
$$

For the case of two parallel links, the mechanism gives 5/4 (see Section 3.1), for Pigou's network it gives 1, see Figure 1.

\footnotetext{
${ }^{4}$ See [26 24] for an excellent exposure of the relevant concepts, the relation among them, as well as for conditions that guarantee their existence.

${ }^{5}$ In [15], Dafermos weakened the orginal definition by [14] to make it closer to the concept of Nash Equilibrium.

${ }^{6}$ See Section 5 for a formal definition.

${ }^{7}$ See for example [16, 4] for examples where equilibria do not exist even for the simplest case of two parallel links and non-decreasing functions.

${ }^{8}$ See [4] for a definition of regular functions.
} 
- For the case of two parallel links with affine cost functions, we describe an optimal 9 mechanism; its engineered Price of Anarchy lies between 1.191 and 1.192 (see Sections 4 and 5). It uses modified cost functions of the form

$$
\hat{\ell}_{e}(x)= \begin{cases}\ell_{e}(x) & \text { for } x \leq r_{e} \text { and } x \geq u_{e} \\ \ell_{e}\left(u_{e}\right) & \text { for } r_{e}<x<u_{e}\end{cases}
$$

The Price of Anarchy is a standard measure to quantify the effect of selfish behavior. There is a vast literature studying the Price of Anarchy for various models of selfish routing and scheduling problems (see [25]). We show that simple coordination mechanisms can reduce the Price of Anarchy for selfish routing games below the $4 / 3$ worst case for networks of parallel links and affine cost functions.

We believe that our arguments extend to more general cost functions, e.g., polynomial cost functions. However, the restriction to parallel links is crucial for our proof. We leave it as a major open problem to prove results for general networks or at least more general networks, e.g., series-parallel networks.

Implementation: We discuss the realization of the modified cost function in a simple traffic scenario where the driving speed on a link is a decreasing function of the flow on the link and hence the transit time is an increasing function. The step function in (3) can be realized by setting a speed limit corresponding to transit time $\ell_{e}\left(u_{e}\right)$ once the flow is above $r_{e}$. The functions in (2) can be approximately realized by access control. In any time unit only $r_{e}$ items are allowed to enter the link. If the usage rate of the link is above $r_{e}$, the queue in front of the link will grow indefinitely and hence transit time will go to infinity.

Related Work: The concept of Coordination Mechanisms was introduced in (the conference version of) [9]. Coordination Mechanisms have been used to improve the Price of Anarchy in scheduling problems for parallel and related machines [9, 19, 22] as well as for unrelated machines [3, 6]; the objective is makespan minimization. Very recently, [10] considered as an objective the weighted sum of completion times. Truthful coordination mechanisms have been studied in [1, 7, 2].

Another very well-studied attempt to cope with selfish behavior is the introduction of taxes (tolls) on the edges of the network in selfish routing games [11, 18, 20, 21, 17, 5]. The disutility of a player is modified and equals her latency plus some toll for every edge that is used in her path. It is well known (see for example [11, 18, 20, 21]) that so-called marginal cost tolls, i.e., $\hat{\ell}_{e}(x)=\ell_{e}(x)+x \ell_{e}^{\prime}(x)$, result in a Nash flow that is equal to the optimum flow for the original cost functions 10 Roughgarden [27] seeks a subnetwork of a given network that has optimal Price of Anarchy for a given demand. [12] studies the question whether tolls can reduce the cost of a Nash equilibrium. They show that for networks with affine latencies, marginal cost pricing does not improve the cost of a flow at Nash equilibrium, as well as that the maximum possible benefit that one can get is no more than that of edge removal.

Discussion: The results of this paper are similar in spirit to the results discussed in the previous paragraph, but also very different. The above papers assume that taxes or tolls are determined with full knowledge of the demand rate $r$. Our coordination mechanisms must a priori decide on the modified latency functions without knowledge of the demand; it must determine the modified functions $\hat{\ell}$ and then an adversary selects the input rate $r$. More importantly, our target objectives are different; we want to minimize the ratio of the modified cost (taking into account the increase of the latencies) over the

\footnotetext{
${ }^{9}$ The lower bound that we provide in Section 5 holds for all deterministic coordination mechanisms that use non-decreasing modified latencies, with respect to both notions of equilibrium described in the previous paragraph.

${ }^{10}$ It is important to observe that although the Nash flow is equal to the optimum flow, its cost with respect to the marginal cost function can be twice as large as its cost with respect to the original cost function. For Pigou's network, the marginal costs are $\hat{\ell}_{1}(x)=2 x$ and $\hat{\ell}_{2}(x)=1$. The cost of a Nash flow of rate $r$ with $r \leq 1 / 2$ is $2 r^{2}$ with respect to marginal costs; the cost of the same flow with respect to the original cost functions is $r^{2}$.
} 
original optimal cost. Our simple strategy presented in Section 3 can be viewed as a generalization of link removal. Removal of a link reduces the capacity of the edge to zero, our simple strategy reduces the capacity to a threshold $r_{e}$. Following [9], we study local mechanisms; the decision of modifying the latency of a link is taken based on the amount of flow that comes through the particular link only 11

\section{Continuous Latency Functions Yield No Improvement}

The network in this section consists of $k$ parallel links connecting $s$ to $t$ and the original latency functions are assumed to be continuous and non-decreasing. We show that substituting them by continuous functions brings no improvement.

Lemma 1. Assume that the original functions $\ell_{e}$ are continuous and non-decreasing. Consider some modified latency functions $\hat{\ell}$ and some rate $r$ for which there is a Nash Equilibrium flow $\hat{f}$ such that the latency function $\hat{\ell}_{i}$ is continuous at $\hat{f}_{i}(r)$ for all $1 \leq i \leq k$. Then $e P o A(r) \geq P o A(r)$.

Proof. It is enough to show that $\hat{C}_{N}(r) \geq C_{N}(r)$. Let $f$ be a Nash flow for rate $r$ and the original cost functions. If $f=\hat{f}$, the claim is obvious. If $\hat{f} \neq f$, there must be a $j$ with $\hat{f}_{j}(r)>f_{j}(r)$. The local continuity of $\hat{\ell}_{i}$ at $\hat{f}_{i}(r)$, implies that $\hat{\ell}_{i}\left(\hat{f}_{i}(r)\right)=\hat{\ell}_{i^{\prime}}\left(\hat{f}_{i^{\prime}}(r)\right)$, for all $i, i^{\prime} \leq k$ such that $\hat{f}_{i}(r), \hat{f}_{i^{\prime}}(r)>0$. Therefore,

$$
\hat{C}_{N}(r)=\hat{C}(\hat{f}(r))=\sum_{i=1}^{k} \hat{f}_{i}(r) \hat{\ell}_{i}\left(\hat{f}_{i}(r)\right)=r \cdot \hat{\ell}_{j}\left(\hat{f}_{j}(r)\right) \geq r \cdot \ell_{j}\left(\hat{f}_{j}(r)\right) \geq r \cdot \ell_{j}\left(f_{j}(r)\right)
$$

since $\hat{\ell}_{j}(x) \geq \ell_{j}(x)$ for all $x$ and $\ell_{j}$ is non-decreasing. Since $f$ is a Nash flow we have $\ell_{i}\left(f_{i}(r)\right) \leq \ell_{j}\left(f_{j}(r)\right)$ for any $i$ with $f_{i}(r)>0$. Thus

$$
C_{N}(r)=\sum_{i=1}^{k} f_{i}(r) \ell_{i}\left(f_{i}(r)\right) \leq r \cdot \ell_{j}\left(f_{j}(r)\right)
$$

\section{A Simple Coordination Mechanism}

Let $\ell_{i}(x)=a_{i} x+b_{i}=\left(x+\gamma_{i}\right) / \lambda_{i}$ be the latency function of the $i$-th link, $1 \leq i \leq k$. We call $\lambda_{i}$ the efficiency of the link. We order the links in order of increasing $b$-value and assume $0 \leq b_{1}<b_{2}<\ldots<b_{k}$ as two links with the same $b$-value may be combined (by adding their efficiencies). We say that a link is used if it carries positive flow. We may assume $a_{i}>0$ for all $i<k$; if $a_{i}=0$, links $i+1$ and higher will never be used. The following theorem summarizes some basic facts about optimal flows and Nash flows; it is proved by straightforward calculations 12 We state the theorem for the case that $a_{k}$ is positive. The theorem is readily extended to the case $a_{k}=0$ by letting $a_{k}$ go to zero and determining the limit values. We will only use the theorem in situations, where $a_{k}>0$.

\footnotetext{
${ }^{11}$ It is not hard to see that, similarly to the case where the demand is known, using global flow information (at least for the case of parallel links) can lead to mechanisms with $e P o A=1$. We would like to thank Nicolás Stier Moses for making us emphasizing that distinction.

${ }^{12}$ In a Nash flow all used links have the same latency. Thus, if $j$ links are used at rate $r$ and $f_{i}^{N}$ is the flow on the $i$-th link, then $a_{1} f_{1}^{N}+b_{1}=\ldots=a_{j} f_{j}^{N}+b_{j} \leq b_{j+1}$ and $r=f_{1}^{N}+\ldots+f_{j}^{N}$. The values for $r_{j}$ and $f_{i}^{N}$ follow from this. Similarly, in an optimal flow all used links have the same marginal costs.
} 
Theorem 1. Let $0 \leq b_{1}<b_{2}<\ldots<b_{k}$ and $\lambda_{i} \geq 0$ for all $i$. Let $\Lambda_{j}=\sum_{i \leq j} \lambda_{i}$ and $\Gamma_{j}=\sum_{i \leq j} \gamma_{j}$. Consider a fixed rate $r$ and let $f_{i}^{*}$ and $f_{i}^{N}, 1 \leq i \leq k$, be the optimal flow and the Nash flow for rate $r$ respectively. Let

$$
r_{j}=\sum_{1 \leq i<j}\left(b_{j}-b_{i}\right) \lambda_{i}=\sum_{1 \leq i<j}\left(b_{i+1}-b_{i}\right) \Lambda_{i} .
$$

Then

(a) $\Gamma_{j}+r_{j}=b_{j} \Lambda_{j}$ and $\Gamma_{j-1}+r_{j}=b_{j} \Lambda_{j-1}$.

(b) If Nash uses exactly $j$ links at rate $r$ then

$$
r_{j} \leq r \leq r_{j+1}, \quad f_{i}^{N}=\frac{r \lambda_{i}}{\Lambda_{j}}+\delta_{i}, \quad \text { where } \delta_{i}=\frac{\Gamma_{j} \lambda_{i}}{\Lambda_{j}}-\gamma_{i}, \quad \text { and } \quad C_{N}(r)=\frac{1}{\Lambda_{j}}\left(r^{2}+\Gamma_{j} r\right) .
$$

(c) If Opt uses exactly $j$ links at rate $r$ then

$$
\frac{r_{j}}{2} \leq r \leq \frac{r_{j+1}}{2}, \quad f_{i}^{*}=\frac{r \lambda_{i}}{\Lambda_{j}}+\delta_{i} / 2, \quad \text { where } \delta_{i}=\frac{\Gamma_{j} \lambda_{i}}{\Lambda_{j}}-\gamma_{i},
$$

and

$$
C_{\text {opt }}(r)=\frac{1}{\Lambda_{j}}\left(r^{2}+\Gamma_{j} r\right)-\sum_{i \leq j} \frac{\delta_{i}^{2}}{4 \lambda_{i}}=\frac{1}{\Lambda_{j}}\left(r^{2}+\Gamma_{j} r\right)-C_{j}, \text { where } C_{j}=\left(\sum_{i=1}^{h} \sum_{h=i}^{j}\left(b_{h}-b_{i}\right)^{2} \lambda_{h} \lambda_{i}\right) /\left(4 \Lambda_{j}\right) .
$$

(d) If $s<r$ and Opt uses exactly $j$ links at $s$ and $r$ then

$$
C_{\text {opt }}(r)=C_{\text {opt }}(s)+\frac{1}{\Lambda_{j}}\left((r-s)^{2}+\left(\Gamma_{j}+2 s\right)(r-s)\right) .
$$

(e) If $s<r$ and Nash uses exactly $j$ links at $s$ and $r$ then

$$
C_{N}(r)=C_{N}(s)+\frac{1}{\Lambda_{j}}\left((r-s)^{2}+\left(\Gamma_{j}+2 s\right)(r-s)\right) .
$$

We next define our simple coordination mechanism. In the case of $k$ links, it is governed by parameters $R_{1}, R_{2}, \ldots, R_{k-1} ; R_{i} \geq 2$ for all $i$. We call the $j$-th link super-efficient (with respect to parameters $R_{1}$ to $R_{k-1}$ ) if $\lambda_{j}>R_{j-1} \Lambda_{j-1}$. In Pigou's network (see Figure 1), the second link is super-efficient for any choice of $R_{1}$ since $\lambda_{2}=\infty$ and $\Lambda_{1}=\lambda_{1}=1$. Super-efficient links are the cause of high Price of Anarchy. Observe that Opt starts using the $j$-th link at rate $r_{j} / 2$ and Nash starts using it at rate $r_{j}$. If the $j$-th link is super-efficient, Opt will send a significant fraction of the total flow across the $j$-th link and this will result in a high Price of Anarchy. Our coordination mechanism induces the Nash flow to use super-efficient links earlier. The latency functions $\hat{\ell}_{i}$ are defined as follows: $\hat{\ell}_{i}=\ell_{i}$ if there is no super-efficient link $j>i$; in particular the latency function of the highest link ( $=$ link $k$ ) is unchanged. Otherwise, we choose a threshold value $T_{i}$ (see below) and set $\hat{\ell}_{i}(x)=\ell_{i}(x)$ for $x \leq T_{i}$ and $\hat{\ell}_{i}(x)=\infty$ for $x>T_{i}$. The threshold values are chosen so that the following behavior results. We call this behavior modified Nash (MN).

Assume that Opt uses $h$ links, i.e., $r_{h} / 2 \leq r \leq r_{h+1} / 2$. If $\lambda_{i+1} \leq R_{i} \Lambda_{i}$ for all $i, 1 \leq i<h$, MN behaves like Nash. Otherwise, let $j$ be minimal such that link $j+1$ is super-efficient; MN changes its behavior at rate $r_{j+1} / 2$. More precisely, it freezes the flow across the first $j$ links at their current values when the total flow is equal to $r_{j+1} / 2$ and routes any additional flow across links $j+1$ to $k$. The thresholds for the lower links are chosen in such a way that this freezing effect takes place. The additional flow is routed by using the strategy recursively. In other words, let $j_{1}+1, \ldots, j_{t}+1$ be the indices of the super-efficient links. Then MN changes behavior at rates $r_{j_{i}+1} / 2$. At this rate the flow across links 1 to $j_{i}$ is frozen and additional flow is routed across the higher links. 
We use $C_{M N}(r)=\hat{C}_{N}^{R_{1}, \ldots, R_{k-1}}(r)$ to denote the cost of MN at rate $r$ when operated with parameters $R_{1}$ to $R_{k-1}$. Then $e P o A(r)=C_{M N}(r) / C_{o p t}(r)$. For the analysis of MN we use the following strategy. We first investigate the benign case when there is no super-efficient link. In the benign case, MN behaves like Nash and the worst case bound of $4 / 3$ on the PoA can never be attained. More precisely, we will exhibit a function $B\left(R_{1}, \ldots, R_{k-1}\right)$ which is smaller than $4 / 3$ for all choices of the $R_{i}$ 's and will prove $C_{M N}(r) \leq B\left(R_{1}, \ldots, R_{k-1}\right) C_{o p t}(r)$. We then investigate the non-benign case. We will derive a recurrence relation for

$$
e P o A\left(R_{1}, \ldots, R_{k-1}\right)=\max _{r} \frac{\hat{C}_{N}^{R_{1}, \ldots, R_{k-1}}(r)}{C_{\text {opt }}(r)} .
$$

In the case of a single link, i.e., $k=1, \mathrm{MN}$ behaves like Nash which in turn is equal to Opt. Thus $e P o A()=1$. The coming subsections are devoted to the analysis of two links and more than two links, respectively.

\subsection{Two Links}

The modified algorithm is determined by a parameter $R \geq 2$. If $\lambda_{2} \leq R \lambda_{1}$, modified Nash is identical to Nash. If $\lambda_{2}>R \lambda_{1}$, the modified algorithm freezes the flow across the first link at $r_{2} / 2$ once it reaches this level, i.e., $\hat{\ell}_{1}(x)=\ell_{1}(x)$ for $x \leq r_{2} / 2$ and $\hat{\ell}_{1}(x)=\infty$ for $x>r_{2} / 213$

Theorem 2. For the case of two links, $e P o A \leq \max \{1+1 / R,(4+4 R) /(4+3 R)\}$. In particular ePoA= $5 / 4$ for $R=4$.

Proof. Consider first the benign case $\lambda_{2} \leq R \Lambda_{1}$. There are three regimes: for $r \leq r_{2} / 2$, Opt and Nash behave identically. For $r_{2} / 2 \leq r \leq r_{2}$, Opt uses both links and Nash uses only the first link, and for $r \geq r_{2}$, Opt and Nash use both links. PoA $(r)$ is increasing for $r \leq r_{2}$ and decreasing for $r \geq r_{2}$. The worst case is at $r=r_{2}$. Then $\operatorname{PoA}\left(r_{2}\right)=C_{N}\left(r_{2}\right) / C_{\text {opt }}\left(r_{2}\right)=C_{N}\left(r_{2}\right) /\left(C_{N}\left(r_{2}\right)-C_{2}\right)=1 /\left(1-C_{2} / C_{N}\left(r_{2}\right)\right)$. We upper-bound $C_{2} / C_{N}\left(r_{2}\right)$. Recall that $r_{2}=\left(b_{2}-b_{1}\right) \lambda_{1}, r_{2}+\Gamma_{1}=b_{2} \lambda_{1}$ and $C_{N}\left(r_{2}\right)=1 / \lambda_{1}\left(r_{2}^{2}+\Gamma_{1} r_{2}\right)$. We obtain

$$
\frac{C_{2}}{C_{N}\left(r_{2}\right)}=\frac{\left(b_{2}-b_{1}\right)^{2} \lambda_{1} \lambda_{2}}{4 \Lambda_{2}\left(1 / \lambda_{1}\right)\left(r_{2}^{2}+\gamma_{1} r_{2}\right)}=\frac{\left(b_{2}-b_{1}\right)^{2} \lambda_{1} \lambda_{2}}{4 \Lambda_{2}\left(1 / \lambda_{1}\right)\left(b_{2}-b_{1}\right) \lambda_{1} b_{2} \lambda_{1}} \leq \frac{\lambda_{2}}{4 \Lambda_{2}} \leq \frac{1}{4(1+1 / R)} .
$$

Thus $\operatorname{PoA}(r) \leq B(R):=\frac{1}{1-\frac{R}{4(R+1)}}=(4+4 R) /(4+3 R)$.

We come to the case $\lambda_{2}>R \Lambda_{1}$ : There are two regimes: for $r \leq r_{2} / 2$, Opt and MN behave identically. For $r>r_{2} / 2$, Opt uses both links and MN routes $r_{2} / 2$ over the first link and $r-r_{2} / 2$ over the second link. Thus for $r \geq r_{2} / 2$ :

$$
e P o A(r)=\frac{C_{M N}(r)}{C_{o p t}(r)}=\frac{C_{o p t}\left(r_{2} / 2\right)+\frac{\left(r-r_{2} / 2\right)^{2}}{\lambda_{2}}+b_{2}\left(r-r_{2} / 2\right)}{C_{o p t}\left(r_{2} / 2\right)+\frac{\left(r-r_{2} / 2\right)^{2}}{\Lambda_{2}}+b_{2}\left(r-r_{2} / 2\right)} \leq \frac{\Lambda_{2}}{\lambda_{2}} \leq 1+1 / R .
$$

\subsection{Many Links}

As already mentioned, we distinguish cases. We first study the benign case $\lambda_{i+1} \leq R_{i} \Lambda_{i}$ for all $i, 1 \leq i<k$, and then deal with the non-benign case.

\footnotetext{
${ }^{13}$ In Pigou's network we have $\ell_{1}(x)=x$ and $\ell_{2}(x)=1$. Thus $\lambda_{2}=\infty$. The modified cost functions are $\hat{\ell}_{2}(x)=\ell_{2}(x)$ and $\hat{\ell}_{1}(x)=x$ for $x \leq r_{2} / 2=1 / 2$ and $\hat{\ell}_{1}(x)=\infty$ for $x>1 / 2$. The Nash flow with respect to the modified cost function is identical to the optimum flow in the original network and $\hat{C}_{N}\left(f^{*}\right)=C\left(f^{*}\right)$. Thus $e P o A=1$ for Pigou's network.
} 
The Benign Case: We assume $\lambda_{i+1} \leq R_{i} \Lambda_{i}$ for all $i, 1 \leq i<k$. Then MN behaves like Nash. We will show $e P o A \leq B\left(R_{1}, \ldots, R_{k-1}\right)<4 / 3$; here $B$ stands for benign case or base case. Our proof strategy is as follows; we will first show (Lemma2) that for the $i$-th link the ratio of Nash flow to optimal flow is bounded by $2 \Lambda_{k} /\left(\Lambda_{i}+\Lambda_{k}\right)$. This ratio is never more than two; in the benign case, it is bounded away from two. We will then use this fact to derive a bound on the Price of Anarchy (Lemma 4).

Lemma 2. Let $h$ be the number of links that Opt is using. Then

$$
\frac{f_{i}^{N}}{f_{i}^{*}} \leq \frac{2 \Lambda_{h}}{\Lambda_{i}+\Lambda_{h}}
$$

for $i \leq h$. If $\lambda_{i^{\prime}+1} \leq R_{i^{\prime}} \Lambda_{i^{\prime}}$ for all $i^{\prime}$, then

$$
\frac{2 \Lambda_{h}}{\Lambda_{i}+\Lambda_{h}} \leq \frac{2 P}{P+1}
$$

where $P:=\prod_{1 \leq i<k}\left(1+R_{i}\right)$.

Proof. Let $j$ be the number of links that Nash is using. For $i>j$, the Nash flow on the $i$-th link is zero and the claim is obvious. For $i \leq j$, we can write the Nash and the optimal flow through link $i$ as

$$
f_{i}^{N}=r \lambda_{i} / \Lambda_{j}+\left(\Gamma_{j} \lambda_{i} / \Lambda_{j}-\gamma_{i}\right) \quad \text { and } \quad f_{i}^{*}=r \lambda_{i} / \Lambda_{h}+\left(\Gamma_{h} \lambda_{i} / \Lambda_{h}-\gamma_{i}\right) / 2 .
$$

Therefore their ratio as a function of $r$ is

$$
F(r)=\frac{f_{i}^{N}}{f_{i}^{*}}=\frac{\Lambda_{h}}{\Lambda_{j}} \cdot \frac{2 r+2 \Gamma_{j}-2 b_{i} \Lambda_{j}}{2 r+\Gamma_{h}-b_{i} \Lambda_{h}}
$$

The sign of the derivative $F^{\prime}(r)$ is equal to the sign of $\Gamma_{h}-b_{i} \Lambda_{h}-2 \Gamma_{j}+2 b_{i} \Lambda_{j}$ and hence constant. Thus $F(r)$ attains its maximum either for $r_{j}$ or for $r_{j+1}$. We have

$$
\begin{aligned}
F\left(r_{j+1}\right) & \leq \frac{\Lambda_{h}}{\Lambda_{j}} \cdot \frac{2 r_{j+1}+2 \Gamma_{j}-2 b_{i} \Lambda_{j}}{2 r_{j+1}+\Gamma_{h}-b_{i} \Lambda_{h}}=\frac{\Lambda_{h}}{\Lambda_{j}} \cdot \frac{2\left(b_{j+1}-b_{i}\right) \Lambda_{j}}{2 b_{j+1} \Lambda_{j+1}-2 \Gamma_{j+1}+\Gamma_{h}-b_{i} \Lambda_{h}} \\
& =\frac{2\left(b_{j+1}-b_{i}\right) \Lambda_{h}}{\sum_{g \leq j+1}\left(2 b_{j+1}-2 b_{g}\right) \lambda_{g}+\sum_{g \leq h}\left(b_{g}-b_{i}\right) \lambda_{g}}=\frac{2\left(b_{j+1}-b_{i}\right) \Lambda_{h}}{\sum_{g \leq j}\left(2 b_{j+1}-b_{g}-b_{i}\right) \lambda_{g}+\sum_{j<g \leq h}\left(b_{g}-b_{i}\right) \lambda_{g}} \\
& =\frac{2\left(b_{j+1}-b_{i}\right) \Lambda_{h}}{\sum_{g \leq i}\left(2 b_{j+1}-b_{g}-b_{i}\right) \lambda_{g}+\sum_{i<g \leq j}\left(2 b_{j+1}-b_{g}-b_{i}\right) \lambda_{g}+\sum_{j<g \leq h}\left(b_{g}-b_{i}\right) \lambda_{g}} \\
& \leq \frac{2\left(b_{j+1}-b_{i}\right) \Lambda_{h}}{\sum_{g \leq i} 2\left(b_{j+1}-b_{i}\right) \lambda_{g}+\sum_{i<g \leq h}\left(b_{j+1}-b_{i}\right) \lambda_{g}} \\
& =\frac{2 \Lambda_{h}}{\sum_{g \leq i} 2 \lambda_{g}+\sum_{i<g \leq h} \lambda_{g}}=\frac{2 \Lambda_{h}}{\Lambda_{i}+\Lambda_{h}}
\end{aligned}
$$

and

$$
\begin{aligned}
F\left(r_{j}\right) & \leq \frac{\Lambda_{h}}{\Lambda_{j}} \cdot \frac{2 r_{j}+2 \Gamma_{j}-2 b_{i} \Lambda_{j}}{2 r_{j}+\Gamma_{h}-b_{i} \Lambda_{h}}=\frac{\Lambda_{h}}{\Lambda_{j}} \cdot \frac{2\left(b_{j}-b_{i}\right) \Lambda_{j}}{2 b_{j} \Lambda_{j}-2 \Gamma_{j}+\Gamma_{h}-b_{i} \Lambda_{h}} \\
& =\frac{2\left(b_{j}-b_{i}\right) \Lambda_{h}}{\sum_{g \leq j}\left(2 b_{j}-2 b_{g}\right) \lambda_{g}+\sum_{g \leq h}\left(b_{g}-b_{i}\right) \lambda_{g}}=\frac{2\left(b_{j}-b_{i}\right) \Lambda_{h}}{\sum_{g \leq j}\left(2 b_{j}-b_{g}-b_{i}\right) \lambda_{g}+\sum_{j<g \leq h}\left(b_{g}-b_{i}\right) \lambda_{g}} \\
& =\frac{2\left(b_{j}-b_{i}\right) \Lambda_{h}}{\sum_{g \leq i}\left(2 b_{j}-b_{g}-b_{i}\right) \lambda_{g}+\sum_{i<g \leq j}\left(2 b_{j}-b_{g}-b_{i}\right) \lambda_{g}+\sum_{j<g \leq h}\left(b_{g}-b_{i}\right) \lambda_{g}} \\
& \leq \frac{2\left(b_{j}-b_{i}\right) \Lambda_{h}}{\sum_{g \leq i} 2\left(b_{j}-b_{i}\right) \lambda_{g}+\sum_{i<g \leq h}\left(b_{j}-b_{i}\right) \lambda_{g}}=\frac{2 \Lambda_{h}}{\sum_{g \leq i} 2 \lambda_{g}+\sum_{i<g \leq h} \lambda_{g}}=\frac{2 \Lambda_{h}}{\Lambda_{i}+\Lambda_{h}} .
\end{aligned}
$$
$P \Lambda_{i}$.

If $\lambda_{i^{\prime}+1} \leq R_{i^{\prime}} \Lambda_{i^{\prime}}$ for all $i^{\prime}$, then $\Lambda_{i^{\prime}+1}=\lambda_{i^{\prime}+1}+\Lambda_{i^{\prime}} \leq\left(1+R_{i^{\prime}}\right) \Lambda_{i^{\prime}}$ for all $i^{\prime}$ and hence $\Lambda_{h} \leq \Lambda_{k} \leq$ 
Lemma 3. For any positive reals $\mu, \alpha$, and $\beta$ with $1 \leq \mu \leq 2$ and $\alpha / \beta \leq \mu, \beta \alpha \leq \frac{\mu-1}{\mu^{2}} \alpha^{2}+\beta^{2}$.

Proof. We may assume $\beta \geq 0$. If $\beta=0$, there is nothing to show. So assume $\beta>0$ and let $\alpha / \beta=\delta \mu$ for some $\delta \leq 1$. We need to show (divide the target inequality by $\left.\beta^{2}\right) \delta \mu \leq(\mu-1) \delta^{2}+1$ or equivalently $\mu \delta(1-\delta) \leq(1-\delta)(1+\delta)$. This inequality holds for $\delta \leq 1$ and $\mu \leq 2$.

Lemma 4. If $f_{i}^{N} / f_{i}^{*} \leq \mu \leq 2$ for all $i$, then $P o A \leq \mu^{2} /\left(\mu^{2}-\mu+1\right)$. If $\lambda_{j+1} \leq R_{j} \Lambda_{j}$ for all $j$, then

$$
P O A \leq B\left(R_{1}, \ldots, R_{k-1}\right):=\frac{4 P^{2}}{3 P^{2}+1},
$$

where $P:=\prod_{1 \leq i<k}\left(1+R_{i}\right)$.

Proof. Assume that Nash uses $j$ links and let $L$ be the common latency of the links used by Nash. Then $L=a_{i} f_{i}^{N}+b_{i}$ for $i \leq j$ and $L \leq b_{i}=a_{i} f_{i}^{N}+b_{i}$ for $i>j$. Thus, by use of Lemma 3 ,

$$
\begin{aligned}
C_{N}(r) & =L r=\sum_{i} L f_{i}^{*} \leq \sum_{i}\left(a_{i} f_{i}^{N}+b_{i}\right) f_{i}^{*} \leq \frac{\mu-1}{\mu^{2}} \sum_{i} a_{i}\left(f_{i}^{N}\right)^{2}+\sum_{i}\left(a_{i}\left(f_{i}^{*}\right)^{2}+b_{i} f_{i}^{*}\right) \\
& \leq \frac{\mu-1}{\mu^{2}} C_{N}(r)+C_{\text {opt }}(r)
\end{aligned}
$$

and hence $P o A \leq \mu^{2} /\left(\mu^{2}-\mu+1\right)$. If $\lambda_{j+1} \leq R_{j} \Lambda_{j}$ for all $j$, employing Lemma 2, we may use $\mu=$ $2 P /(P+1)$ and obtain $P o A \leq 4 P^{2} /\left(3 P^{2}+1\right)$.

The General Case: We come to the case where $\lambda_{i+1} \geq R_{i} \Lambda_{i}$ for some $i$. Let $j$ be the smallest such $i$. For $r \leq r_{j+1} / 2$, MN and Opt use only links 1 to $j$ and we are in the benign case. Hence $e P o A$ is bounded by $B\left(R_{1}, \ldots, R_{j-1}\right)<4 / 3$. Assume now that $r>r_{j+1 / 2}$. MN routes the flow exceeding $r_{j+1} / 2$ exclusively on higher links.

\section{Lemma 5. MN does not use links before Opt.}

Proof. This is trivially true for the $j+1$-st link. Consider any $h>j+1$. MN starts to use link $h$ at $s_{h}=r_{j+1} / 2+\sum_{j+1 \leq i<h}\left(b_{i+1}-b_{i}\right)\left(\Lambda_{i}-\Lambda_{j}\right)$ and Opt starts to use it at $r_{h} / 2=r_{j+1} / 2+\sum_{j+1 \leq i<h}\left(b_{i+1}-\right.$ $\left.b_{i}\right) \Lambda_{i} / 2$. We have $s_{h} \geq r_{h} / 2$ since $\Lambda_{i}-\Lambda_{j} \geq \Lambda_{i} / 2$ for $i>j$.

We need to bound the cost of $\mathrm{MN}$ in terms of the cost of Opt. In order to do so, we introduce an intermediate flow Mopt (modified optimum) that we can readily relate to $\mathrm{MN}$ and to Opt. Mopt uses links 1 to $j$ to route $r_{j+1} / 2$ and routes $f=r-r_{j+1} / 2$ optimally across links $j+1$ to $k$. Let $f_{i}^{*}$ and $f_{i}^{m}$ be the optimal flows and the flows of Mopt, respectively, at rate $r$. Let $r_{s}=\sum_{i \leq j} f_{i}^{*} \geq r_{j+1} / 2$ be the total flow routed across the first $j$ links in the optimal flow (the subscript $s$ stands for small) and let

$$
t=\frac{r-r_{j+1} / 2}{r-r_{s}}
$$

We will show $t \leq 1+1 / R_{j}$ below. We next relate the cost of Mopt on links $j+1$ to $k$ to the cost of Opt on these links. To this end we scale the optimal flow on these links by a factor of $t$, i.e., we consider the following flow across links $j+1$ to $k$ : on link $i, j+1 \leq i \leq k$, it routes $t \cdot f_{i}^{*}$. The total flow on the high links, i.e., links $j+1$ to $k$, is $r-r_{j+1} / 2$ and hence Mopt incurs at most the cost of this flow on its high links. Thus

$$
\sum_{i>j} \ell_{i}\left(f_{i}^{m}\right) f_{i}^{m} \leq \sum_{i>j} \ell_{i}\left(t f_{i}^{*}\right) t f_{i}^{*} \leq t^{2}\left(\sum_{i>j} \ell_{i}\left(f_{i}^{*}\right) f_{i}^{*}\right) .
$$


The cost of $\mathrm{MN}$ on the high links is at most $e P o A\left(R_{j+1}, \ldots, R_{k-1}\right)$ times this cost by the induction hypothesis. We can now bound the cost of $\mathrm{MN}$ as follows:

$$
\begin{aligned}
C_{M N}(r) & =C_{N}\left(r_{j+1} / 2\right)+C_{M N}(\text { flow } f \text { across links } j+1 \text { to } k) \\
& \leq B\left(R_{1}, \ldots, R_{j-1}\right) C_{o p t}\left(r_{j+1} / 2\right)+t^{2} e P o A\left(R_{j+1}, \ldots, R_{k-1}\right)\left(\sum_{i>j} \ell_{i}\left(f_{i}^{*}\right) f_{i}^{*}\right) \\
& \leq B\left(R_{1}, \ldots, R_{j-1}\right)\left(\sum_{i \leq j} \ell_{i}\left(f_{i}^{*}\right) f_{i}^{*}\right)+t^{2} e P o A\left(R_{j+1}, \ldots, R_{k-1}\right)\left(\sum_{i>j} \ell_{i}\left(f_{i}^{*}\right) f_{i}^{*}\right) \\
& \leq \max \left\{B\left(R_{1}, \ldots, R_{j-1}\right), t^{2} e P o A\left(R_{j+1}, \ldots, R_{k-1}\right)\right\} C_{o p t}(r)
\end{aligned}
$$

Lemma 6. $t \leq 1+1 / R_{j}$, where $j$ is the smallest $i$ for which $\lambda_{i+1} \geq R_{i} \Lambda_{i}$.

Proof. Assume that Opt uses $h$ links where $j+1 \leq h \leq k$. Then $r_{h} / 2 \leq r \leq r_{h+1} / 2$. Let $r=r_{h} / 2+\delta$. According to Theorem $1 f_{i}^{*}=r \lambda_{i} / \Lambda_{h}+\left(\Gamma_{h} \lambda_{i} / \Lambda_{h}-\gamma_{i}\right) / 2$ and hence

$$
r_{s}=\left(\frac{r_{h}}{2}+\delta\right) \frac{\Lambda_{j}}{\Lambda_{h}}+\frac{1}{2}\left(\frac{\Gamma_{h} \Lambda_{j}}{\Lambda_{h}}-\Gamma_{j}\right)
$$

Since $\Gamma_{h}+r_{h}=b_{h} \Lambda_{h}$ and $\Gamma_{j}+r_{j}=b_{j} \Lambda_{j}$ (see Theorem 1), this simplifies to

$$
r_{s}=\frac{\Lambda_{j} \delta}{\Lambda_{h}}+\frac{b_{h} \Lambda_{h}-\Gamma_{h}}{2} \frac{\Lambda_{j}}{\Lambda_{h}}+\frac{1}{2}\left(\frac{\Gamma_{h} \Lambda_{j}}{\Lambda_{h}}-b_{j} \Lambda_{j}+r_{j}\right)=\frac{\Lambda_{j} \delta}{\Lambda_{h}}+\frac{1}{2}\left(\left(b_{h}-b_{j}\right) \Lambda_{j}+r_{j}\right)=\frac{\Lambda_{j} \delta}{\Lambda_{h}}+r_{s}^{*},
$$

where $r_{s}^{*}=\frac{1}{2}\left(\left(b_{h}-b_{j}\right) \Lambda_{j}+r_{j}\right)$. We can now bound $t$.

$$
t=\frac{r-r_{j+1} / 2}{r-r_{s}}=\frac{r_{h} / 2+\delta-r_{j+1} / 2}{r_{h} / 2+\delta-r_{s}^{*}-\Lambda_{j} \delta / \Lambda_{h}} \leq \max \left\{\frac{r_{h} / 2-r_{j+1} / 2}{r_{h} / 2-r_{s}^{*}}, \frac{1}{1-\frac{\Lambda_{j}}{\Lambda_{h}}}\right\} .
$$

Next observe that

$$
\begin{aligned}
\frac{r_{h} / 2-r_{j+1} / 2}{r_{h} / 2-r_{s}^{*}} & =\frac{\sum_{j+1 \leq i<h}\left(b_{i+1}-b_{i}\right) \Lambda_{i}}{\left(\sum_{j \leq i<h}\left(b_{i+1}-b_{i}\right) \Lambda_{i}\right)-\left(b_{h}-b_{j}\right) \Lambda_{j}}=\frac{\sum_{j+1 \leq i<h}\left(b_{i+1}-b_{i}\right) \Lambda_{i}}{\sum_{j+1 \leq i<h}\left(b_{i+1}-b_{i}\right)\left(\Lambda_{i}-\Lambda_{j}\right)} \\
& \leq \max _{j+1 \leq i<h} \frac{\Lambda_{i}}{\Lambda_{i}-\Lambda_{j}}=\frac{\Lambda_{j+1}}{\Lambda_{j+1}-\Lambda_{j}}=\frac{\Lambda_{j}+\lambda_{j+1}}{\lambda_{j+1}} \leq 1+\frac{1}{R_{j}} .
\end{aligned}
$$

The second term in the upper bound for $t$ is also bounded by this quantity.

We summarize the discussion.

Lemma 7. For every $k$ and every $j$ with $1 \leq j<k$. If $\lambda_{j+1}>R_{j} \Lambda_{j}$ and $\lambda_{i+1} \leq R_{i} \Lambda_{i}$ for $i<j$, then

$$
e P o A\left(R_{1}, \ldots, R_{k-1}\right) \leq \max \left\{B\left(R_{1}, \ldots, R_{j-1}\right),\left(1+\frac{1}{R_{j}}\right)^{2} e P o A\left(R_{j+1}, \ldots, R_{k-1}\right)\right\} .
$$

We are now ready for our main theorem.

Theorem 3. For any $k$, there is a choice of the parameters $R_{1}$ to $R_{k-1}$ such that the engineered Price of Anarchy with these parameters is strictly less than $4 / 3$. 
Proof. We will show $e P o A\left(R_{i}, \ldots, R_{k-1}\right)<4 / 3$ by downward induction on $i$, i.e., we will define $R_{k-1}$, $R_{k-2}$, down to $R_{1}$ in this order. For $i=k$, we have $e P o A()=1<4 / 3$.

We now come to the induction step. We have already defined $R_{k-1}$ down to $R_{i+1}$ and now define $R_{i}$. We have

$$
e P o A\left(R_{i}, \ldots, R_{k-1}\right) \leq \max \left\{\begin{array}{l}
B\left(R_{i}, \ldots, R_{k-1}\right), \\
\max _{j ; i \leq j<k}\left\{B\left(R_{i}, \ldots, R_{j-1}\right),\left(1+\frac{1}{R_{j}}\right)^{2} e P o A\left(R_{j+1}, \ldots, R_{k-1}\right)\right\}
\end{array}\right\},
$$

where the first line covers the benign case and the second line covers the non-benign case (Lemma 7). We now fix $R_{i}$. We have $B\left(R_{i}, \ldots, R_{k-1}\right)<4 / 3$ and $B\left(R_{i}, \ldots, R_{j-1}\right)<4 / 3$ for all $j, i \leq j<k$ by Lemma4 for any choice of $R_{i}$. This completes the induction if the first line defines the maximum. So assume that the second line defines the maximum. We only need to deal with the case $j=i$ in the second line, as the case $j>i$ was already dealt with for larger $i$. The case $j=i$ is handled by choosing $R_{i}$ sufficiently large, i.e., such that $\left(1+1 / R_{i}\right)^{2} e P o A\left(R_{i+1}, \ldots, R_{k-1}\right)<4 / 3$.

Remark 1. Alternatively, we could take $i=k-1$ as the base case. Then, $j=i-1$ in the non-benign case and hence

$$
\begin{aligned}
e P o A\left(R_{k-1}\right) & =\max \left(B\left(R_{k-1}\right), B(),\left(1+\frac{1}{R_{k-1}}\right)^{2} e P o A()\right) \\
& =\max \left(\frac{4\left(1+R_{k-1}\right)^{2}}{3\left(1+R_{k-1}\right)^{2}+1},\left(1+\frac{1}{R_{k-1}}\right)^{2}\right)
\end{aligned}
$$

where the bound on $B\left(R_{k-1}\right)$ comes from Lemma 4 . For $R_{k-1}=7$, the bound becomes $\max ((4 / 3)(64 / 65), 64 / 49)=$ $\max (64 / 65,48 / 49) \cdot 4 / 3$.

\section{An Improved Mechanism for the Case of Two Links}

In this section we present a mechanism which achieves $e P o A=1.192$ for a network that consists of two parallel links. The ratio $C_{N}(r) / C_{\text {opt }}(r)$ is maximized at $r=r_{2}$. At this rate Nash still uses only the first link and Opt uses both links. In order to avoid this maximum ratio (if larger than 1.192), we force MN to use the second link earlier by increasing the latency of the first link after some rate $x_{1}, r_{2} / 2 \leq x_{1} \leq r_{2}$ to a value above $b_{2}$. In the preceding section, we increased the latency to $\infty$. In this way, we avoided a bad ratio at $r_{2}$, but paid a price for very large rates. The idea for the improved construction, is to increase the latency to a finite value. This will avoid the bad ratio, but also allow MN to use both links for large rates. In particular, we obtain the following result.

Theorem 4. There is a mechanism for a network of two parallel links that achieves ePoA $=1.192$.

Proof. Recall (Theorem 2) that the Price of Anarchy is upper bounded by $(4+4 R) /(4+3 R)$ where $R=a_{1} / a_{2}$. Let $R_{0}$ be such that $\left(4+4 R_{0}\right) /\left(4+3 R_{0}\right)=1.192$. Then $R_{0}=96 / 53$. We only need to consider the case $R>R_{0}$. The latency function of the second link is unchanged and the latency function of the first link is changed into

$$
\widehat{\ell}_{1}(x)= \begin{cases}\ell_{1}(x), & x \leq x_{1} \\ \ell_{1}\left(x_{2}\right), & x_{1}<x \leq x_{2} \\ \ell_{1}(x) & x>x_{2}\end{cases}
$$

where $x_{1}$ and $x_{2}$ satisfy $r_{2} / 2 \leq x_{1} \leq r_{2} \leq x_{2}$ and will be fixed later. In words, when either the flow in the first link does not exceed $x_{1}$, or is larger than $x_{2}$, the network remains unchanged. However, when 


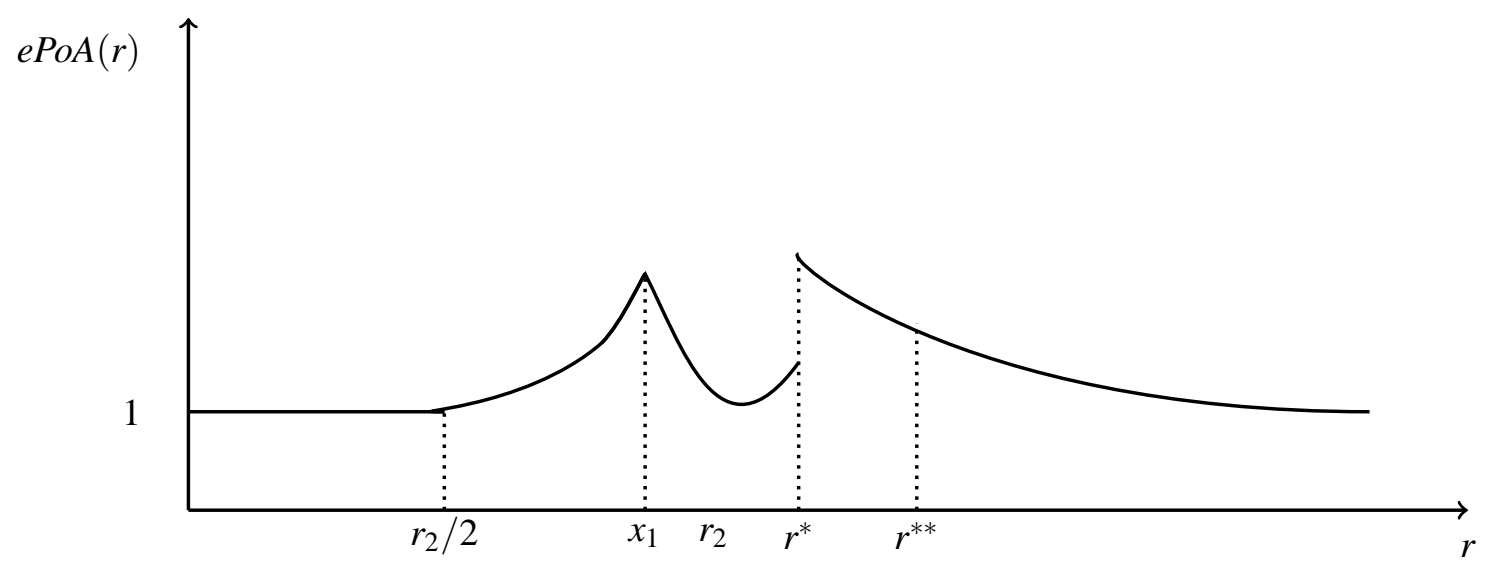

Figure 2: The engineered price of anarchy for the construction of Section 4

the flow in the first link is between these two values, the mechanism increases the latency of this link to $\ell_{1}\left(x_{2}\right)$. Let $r^{*}$ be such that

$$
\ell_{2}\left(r^{*}-x_{1}\right)=\ell_{1}\left(x_{2}\right)
$$

We will fix $x_{1}$ and $x_{2}$ such that $r^{*} \geq r_{2}$.

What is the effect of this modification? For $r \leq r_{2} / 2$, Opt and $\mathrm{MN}$ are the same and $e P o A(r)=1$. For $r_{2} / 2 \leq r \leq x_{1}$, MN behaves like Nash and $e P o A(r)$ increases. At $r=x_{1}$, MN starts to use the second link. MN will route any additional flow on the second link until $r=r^{*}$. At $r=r^{*}$, MN routes $x_{1}$ on the first link and $r^{*}-x_{1}$ on the second link. Beyond $r^{*}$, MN routes additional flow on the first link until the flow on the first link has grown to $x_{2}$. This is the case at $r^{* *}=r^{*}-x_{1}+x_{2}$. For $r \geq r^{* *}$, MN behaves like Nash.

Figure 2]shows the graph of $e P o A(r)$. We have $e P o A(r)=1$ for $r \leq r_{2} / 2$. For $r_{2} / 2 \leq r \leq x_{1}, e P o A(r)$ increases to

$$
e P o A\left(x_{1}\right)=\frac{a_{1} x_{1}^{2}+b_{1} x_{1}}{C_{\text {opt }}\left(x_{1}\right)}
$$

For $x_{1} \leq r \leq r^{*}, e P o A(r)$ is convex. It will first decrease and reach the value one (this assumes that $r^{*}$ is big enough) at the rate where Opt routes $x_{1}$ on the first link; after this rate it will increase again. At $r^{*}$, $e P o A$ has a discontinuity because at $r^{*} \mathrm{MN}$ routes $x_{1}$ on the first link for a cost of $\ell_{1}\left(x_{1}\right) x_{1}$ and at $r^{*}+\varepsilon$ it routes $x_{1}+\varepsilon$ on the first link for a cost of $\ell_{1}\left(x_{2}\right)\left(x_{1}+\varepsilon\right)$. Thus

$$
\lim _{r \rightarrow r_{+}^{*}} \frac{C_{M N}(r)}{C_{o p t}(r)}=\lim _{r \rightarrow r_{+}^{*}} \frac{\ell_{1}\left(x_{2}\right) r}{C_{o p t}(r)}=\frac{\ell_{1}\left(x_{2}\right) r^{*}}{C_{o p t}\left(r^{*}\right)}=\frac{\ell_{2}\left(r^{*}-x_{1}\right) r^{*}}{C_{o p t}\left(r^{*}\right)} .
$$

For $r \geq r^{*}, e P o A(r)$ decreases. Thus

$$
e P o A=\max \left\{\frac{a_{1} x_{1}^{2}+b_{1} x_{1}}{C_{\text {opt }}\left(x_{1}\right)}, \frac{\ell_{2}\left(r^{*}-x_{1}\right) r^{*}}{C_{\text {opt }}\left(r^{*}\right)}\right\} .
$$

It remains to show that $x_{1} \leq r_{2}$ and $r^{*} \geq r_{2}$ can be chosen 14 such that the right-hand side is at most 1.192. By Theorem 1(c),

$$
C_{o p t}(r)=b_{1} r+\frac{a_{1}}{1+R}\left(r^{2}+R r_{2} r-R r_{2}^{2} / 4\right)
$$

\footnotetext{
${ }^{14}$ The optimal choice for $x_{1}$ and $r^{*}$ is such that both terms are equal and as small as possible. We were unable to solve the resulting system explicitly. We will prove in the next section that the mechanism defined by these optimal choices of the parameters $x_{1}$ and $r^{*}$ is optimal.
} 
for $r \geq r_{2} / 2$ and $R=a_{1} / a_{2}$. Also $\ell_{2}\left(r^{*}-x_{1}\right)=a_{2}\left(r^{*}-x_{1}\right)+b_{2}=a_{2}\left(r^{*}-x_{1}\right)+b_{1}+a_{1} r_{2}$.

We first determine the maximum $x_{1} \leq r_{2}$ such that $\left(a_{1} x_{1}^{2}+b_{1} x_{1}\right) / C_{\text {opt }}\left(x_{1}\right) \leq 1.192$ for all $b_{1}$. Since the expression $\left(a_{1} x_{1}^{2}+b_{1} x_{1}\right) / C_{\text {opt }}\left(x_{1}\right)$ is decreasing in $b_{1}$, this $x_{1}$ is determined for $b_{1}=0$. It follows that $\alpha=x_{1} / r_{2}$ is defined by the equation

$$
\frac{4(R+1) \alpha^{2}}{4 \alpha R-R+4 \alpha^{2}}=1.192
$$

For $R \geq R_{0}$, this equation has a unique solution $\alpha_{0} \in[1 / 2,1]$, namely

$$
\alpha_{0}=\frac{1}{2} \cdot \frac{149 R+2 \sqrt{894 R(R+1)}}{125 R-24} .
$$

We turn to the second term in equation (5). For $r^{*}>r_{2}$, it is a decreasing function of $b_{1}$. Substituting $b_{1}=0$ into the second term and setting $\beta=r^{*} / r_{2}$ yields after some computation

$$
e P o A_{2}=\frac{4 \beta(R+1)(\beta-\alpha+R)}{R\left(4 \beta^{2}+4 \beta R-R\right)} .
$$

For fixed $\alpha=\alpha_{0}$ and any $R \geq R_{0}, e P o A_{2}$ is minimized for $\beta=\beta_{0}=\frac{R+\sqrt{R} \sqrt{R+4 \alpha_{0}\left(R-\alpha_{0}\right)}}{4 \alpha_{0}}$. For $R \geq R_{0}$, one can prove $\beta_{0} \geq 1$, as needed. Substituting $\alpha_{0}$ and $\beta_{0}$ into $e P o A_{2}$ yields a function of $R$. It is easy to see, using the derivative, that the maximum value of this function for $R \geq R_{0}$ is at most 1.192.

\section{A Lower Bound for the Case of Two Links}

We prove that the construction of the previous section is optimal among the class of deterministic mechanisms that guarantee the existence of an equilibrium for every rate $r>0$ and that use non-decreasing 15 latency functions. For these mechanisms we show that $e P o A \geq 1.191$.

As in the preceding sections, we use $\hat{\ell}$ to denote the modified latency functions. The use of $R$ throughout this section denotes the ratio of the linear coefficients of the two latency functions of the instance, and should not be confused with its use in the previous sections, were it was a parameter of the mechanism. As mentioned above, we are making two assumptions about the $\hat{\ell}$ 's: an equilibrium flow must exist for every rate $r$, and $\hat{\ell}_{i}$ is non-decreasing, i.e., if $x<x^{\prime}$, then $\hat{\ell}_{i}(x) \leq \hat{\ell}_{i}\left(x^{\prime}\right)$, for $i=1,2$. It is worthwhile to recall the equilibrium conditions for general latency functions (as given by DafermosSparrow [14]): if $(x, y)$ is an equilibrium for rate $r=x+y$, then $\hat{\ell}_{2}\left(y^{\prime}\right) \geq \hat{\ell}_{1}(x)$ for $y^{\prime} \in(y, r]$ (otherwise $y^{\prime}-y$ amount of flow would move from the first link to the second) and $\hat{\ell}_{1}\left(x^{\prime}\right) \geq \hat{\ell}_{2}(y)$ for $x^{\prime} \in(x, r]$ (otherwise, $x^{\prime}-x$ amount of flow would move from the second link to the first). Since we assume our functions to be monotone, the condition $\hat{\ell}_{2}\left(y^{\prime}\right) \geq \hat{\ell}_{1}(x)$ for $y^{\prime} \in(y, r]$ is equivalent to $\liminf _{y^{\prime} \downarrow y} \hat{\ell}_{2}\left(y^{\prime}\right) \geq$ $\hat{\ell}_{1}(x)$ provided that $y<r$ (or equivalently, $x>0$ ). Since we are discussing a network of two parallel links, the latter condition is in turn equivalent to (1).

Theorem 5. The construction of Section 4 is optimal and $e P o A \geq 1.191$.

Proof. We analyze a network with latency functions $\ell_{1}(x)=x$ and $\ell_{2}(x)=x / R+1=(x+R) / R, 2 \leq$ $R \leq 4$, and derive a lower bound as a function of the parameter $R$; the restriction $2 \leq R \leq 4$ will become clear below. In a second step we choose $R$ so as to maximize the lower bound; the optimal choice is $R=R^{*} \approx 2.1$. For $r \leq 1 / 2$, Opt uses one link and $C_{o p t}(r)=r^{2}$, and for $r \geq 1 / 2$, Opt uses two links and $C_{\text {opt }}(r)=\left(r^{2}+R r-R / 4\right) /(1+R) ; C_{\text {opt }}(1)=(3 R+4) /(4 R+4)$ and $C_{N}(1)=1$. Thus $P o A=P o A(1)=$ $(4 R+4) /(3 R+4)$. For $R \geq 2$, we have $P o A(1) \geq 12 / 10=1.2$.

\footnotetext{
${ }^{15}$ It remains open whether similar arguments can be applied for showing the lower bound for non-monotone mechanisms with respect to User Equilibria.
} 
Consider now some modified latency functions $\hat{\ell}_{1}, \hat{\ell}_{2}$, and let $\left(x_{1}, 1-x_{1}\right)$ be an equilibrium flow for rate 1 for the modified network. Let

$$
r^{*}=\inf \left\{r ; \text { there is an equilibrium flow }(x, r-x) \text { for MN with } x>x_{1}\right\} \text {; }
$$

$r^{*}=\infty$ if there is no equilibrium flow $(x, y)$ with $x>x_{1}$. The equilibrium conditions for flow $\left(x_{1}, 1-x_{1}\right)$ imply

$$
\hat{\ell}_{1}\left(x^{\prime}\right) \geq \hat{\ell}_{2}\left(1-x_{1}\right) \geq \ell_{2}\left(1-x_{1}\right) \geq 1 \text { for } x_{1}<x^{\prime} \leq 1 \text {. }
$$

The above definition of $r^{*}$ is a core element of our proof. In Lemma 8 we restrict the domain of $r^{*}$, as well as the range of the modified latencies for efficient mechanisms (those ones with low ePoA), ending up with the lower bound provided in (9). Then in Lemmas 9, 10 we focus on the properties that the equilibria of efficient mechanisms should satisfy. In Lemma 9 , we bound from above the amount of equilibrium flow that uses the first link if the mechanism is efficient, while in Lemma 10 we obtain a second lower bound on the $e P o A$. Finally, in Lemma 11 we summarize the above properties ending up with the lower bound of (11).

Lemma 8. If $\hat{\ell}_{1}\left(x_{1}\right) \geq 1$ or $r^{*}=\infty$ or $r^{*} \leq 1, e P o A \geq 1.2$.

Proof. If $\hat{\ell}_{1}\left(x_{1}\right) \geq 1$, we have $C_{M N}(1) \geq 1$ and hence $e P o A \geq P o A(1) \geq 1.2$.

If $r^{*}=\infty, e P o A(\infty) \geq 1+1 / R$. For $R \leq 4$, this is at least 1.25 .

If $r^{*} \leq 1$, there is an equilibrium flow $(x, y)$ with $x>x_{1}$ and $r=x+y \leq 1$. Then $\hat{\ell}_{1}(x) \geq 1$ by inequality (8). Also $\hat{\ell}_{2}(y) \geq 1$. Thus $C_{M N}(r) \geq 1 \geq r$ and hence $e P o A(r) \geq r / C_{\text {opt }}(r)$. For $r \leq 1$, we have

$$
\frac{r}{C_{\text {opt }}(r)}=\frac{r(1+R)}{r^{2}+R r-R / 4} \geq 1+\frac{R / 4}{r^{2}+R r-R / 4} \geq 1+\frac{R / 4}{1+R-R / 4}=\frac{4+4 R}{4+3 R}=\operatorname{PoA}(1) \geq 1.2 .
$$

In the light of the Lemma above, we proceed under the assumption $\hat{\ell}_{1}\left(x_{1}\right)<1$, and hence $x_{1}<1$, and $1<r^{*}<\infty$. Then $\left(x_{1}, 0\right)$ is an equilibrium flow, since $\hat{\ell}_{1}\left(x_{1}\right)<1 \leq \hat{\ell}_{2}\left(y^{\prime}\right)$ for $0<y^{\prime} \leq x_{1}$. Thus

$$
e P o A\left(x_{1}\right) \geq \frac{x_{1}^{2}}{C_{\text {opt }}\left(x_{1}\right)}
$$

By definition of $r^{*}$, MN routes at most $x_{1}$ on the first link for any rate $r<r^{*}$ and for any $\varepsilon>0$ there is an $r<r^{*}+\varepsilon$ such that $(x, r-x)$ with $x>x_{1}$ is an equilibrium flow for MN.

For $r<r^{*}$, any equilibrium flow $(x, r-x)$ has $x \leq x_{1}$. Thus, for $x^{\prime} \in(x, r] \supseteq\left(x_{1}, r\right], \hat{\ell}_{1}\left(x^{\prime}\right) \geq \hat{\ell}_{2}(r-$ $x) \geq \ell_{2}(r-x) \geq \ell_{2}\left(r-x_{1}\right)$. Since this inequality holds for any $r<r^{*}$, we have

$$
\hat{\ell}_{1}\left(x^{\prime}\right) \geq \ell_{2}\left(r^{*}-x_{1}\right) \text { for } \quad x^{\prime} \in\left(x_{1}, r^{*}\right) \text {. }
$$

For $\varepsilon>0$, let

$$
F_{\varepsilon}=\left\{(x, y) ;(x, y) \text { is an equilibrium flow with } r^{*} \leq x+y \leq r^{*}+\varepsilon \text { and } x>x_{1}\right\} .
$$

Observe that $F_{\varepsilon}$ is non-empty by definition of $r^{*}$.

Lemma 9. If for arbitrarily small $\varepsilon>0$, there is a $(x, y) \in F_{\varepsilon}$ with $x \geq r^{*}$, then $e P o A \geq P o A(1) \geq 1.2$.

Proof. Let $r=x+y$. Then $e P o A \geq r^{2} / C_{\text {opt }}(r)$. Since this inequality holds for arbitrarily small $\varepsilon$,

$$
e P o A \geq\left(r^{*}\right)^{2} / C_{o p t}\left(r^{*}\right) \geq P o A(1)=1.2 .
$$


We proceed under the assumption that there is an $\varepsilon_{0}>0$ such that $F_{\varepsilon_{0}}$ contains no pair $(x, y)$ with $x \geq r^{*}$.

Lemma 10. If for arbitrarily small $\varepsilon \in\left(0, \varepsilon_{0}\right), F_{\varepsilon}$ contains either a pair $\left(x, r^{*}-x_{1}\right)$ or pairs $(x, y)$ and $(u, v)$ with $y \neq v$, then $e P o A \geq \ell_{2}\left(r^{*}-x_{1}\right) r^{*} / C_{\text {opt }}\left(r^{*}\right)$.

Proof. Assume first that $F_{\varepsilon}$ contains a pair $\left(x, r^{*}-x_{1}\right)$ and let $r=x+r^{*}-x_{1}$. Then

$$
C_{M N}(r)=\hat{\ell}_{1}(x) x+\hat{\ell}_{2}\left(r^{*}-x_{1}\right)\left(r^{*}-x_{1}\right) \geq \ell_{2}\left(r^{*}-x_{1}\right) r
$$

since $\hat{\ell}_{1}(x) \geq \ell_{2}\left(r^{*}-x_{1}\right)$ by (10).

Assume next that $F_{\varepsilon}$ contains pairs $(x, y)$ and $(u, v)$ with $y \neq v$. Then $\hat{\ell}_{1}(x) \geq \ell_{2}\left(r^{*}-x_{1}\right)$ and $\hat{\ell}_{1}(u) \geq$ $\ell_{2}\left(r^{*}-x_{1}\right)$ by (10). We may assume, $y>v$. Let $r=x+y$. Since $(u, v)$ is an equilibrium $\hat{\ell}_{2}\left(y^{\prime}\right) \geq \hat{\ell}_{1}(u)$ for $y^{\prime} \in(v, u+v)$ and hence $\hat{\ell}_{2}(y) \geq \hat{\ell}_{1}(u)$. Thus

$$
C_{M N}(r)=\hat{\ell}_{1}(x) x+\hat{\ell}_{2}(y) y \geq \ell_{2}\left(r^{*}-x_{1}\right) r .
$$

We have now shown that $C_{M N}(r) \geq \ell_{2}\left(r^{*}-x_{1}\right) r$ for $r^{\prime}$ s greater than $r^{*}$ and arbitrarily close to $r^{*}$. Thus $e P o A \geq \ell_{2}\left(r^{*}-x_{1}\right) r^{*} / C_{o p t}\left(r^{*}\right)$.

We proceed under the assumption that there is an $\varepsilon_{0}>0$ such that $F_{\varepsilon_{0}}$ contains no pair $(x, y)$ with $x \geq r^{*}$, no pair $\left(x, r^{*}-x_{1}\right)$ and no two pairs with distinct second coordinate. In other words, there is a $y_{0}<r^{*}-x_{1}$ such that all pairs in $F_{\varepsilon_{0}}$ have second coordinate equal to $y_{0}$.

Let $\left(x_{0}, y_{0}\right) \in F_{\varepsilon_{0}}$. Then $y_{0}<r^{*}-x_{1}$. Let $(x, y)$ be an equilibrium for rate $r=\left(r^{*}+x_{1}+y_{0}\right) / 2$. Then $r=\left(2 r^{*}+y_{0}-\left(r^{*}-x_{1}\right)\right) / 2<r^{*}$ and hence $x \leq x_{1}$. Thus $y=r-x \geq r-x_{1}=\left(r^{*}-x_{1}+y_{0}\right) / 2>y_{0}$ and $r-y_{0}>x_{1}$. Consider the pair $\left(r-y_{0}, y_{0}\right)$. Its rate is less than $r^{*}$ and its flow across the first link is $r-y_{0}$ which is larger than $x_{1}$. Thus it is not an equilibrium by the definition of $r^{*}$. Therefore there is either an $x^{\prime \prime} \in\left(r-y_{0}, r\right]$ with $\hat{\ell}_{1}\left(x^{\prime \prime}\right)<\hat{\ell}_{2}\left(y_{0}\right)$ or a $y^{\prime \prime} \in\left(y_{0}, r\right]$ with $\hat{\ell}_{2}\left(y^{\prime \prime}\right)<\hat{\ell}_{1}\left(r-y_{0}\right)$. We now distinguish cases.

Assume the former. Since $(x, y)$ is an equilibrium, we have $\hat{\ell}_{1}\left(x^{\prime}\right) \geq \hat{\ell}_{2}(y)$ for all $x^{\prime} \in(x, x+y]$ and in particular for $x^{\prime \prime}$; observe that $r-y_{0} \geq x$ since $r-x=y>y_{0}$. Thus $\hat{\ell}_{2}\left(y_{0}\right)>\hat{\ell}_{2}(y)$, a contradiction to the monotonicity of $\hat{\ell}_{2}$.

Assume the latter. Since $\left(x_{0}, y_{0}\right)$ is an equilibrium, we have $\hat{\ell}_{2}\left(y^{\prime}\right) \geq \hat{\ell}_{1}\left(x_{0}\right)$ for all $y^{\prime} \in\left(y_{0}, x_{0}+y_{0}\right]$ and in particular for $y^{\prime \prime}$. Thus $\hat{\ell}_{1}\left(r-y_{0}\right)>\hat{\ell}_{1}\left(x_{0}\right)$, a contradiction to the monotonicity of $\hat{\ell}_{1}$; observe that $r-y_{0}<x_{0}$ since $r<r^{*} \leq x_{0}+y_{0}$.

\section{Lemma 11.}

$$
e P o A \geq \min \left\{1.2, \min _{x_{1} \leq 1} \max \left\{\frac{x_{1}^{2}}{C_{o p t}\left(x_{1}\right)}, \min _{r^{*} \geq 1} \frac{\ell_{2}\left(r^{*}-x_{1}\right) r^{*}}{C_{o p t}\left(r^{*}\right)}\right\}\right\} .
$$

Proof. If $x_{1} \geq 1$ or $r^{*} \leq 1$ or $r^{*}=\infty$, we have $e P o A \geq 1.2$. So assume $x_{1}<1$ and $1<r^{*}<\infty$. The argument preceding this Lemma shows that the hypothesis of either Lemma 9 or 10 is satisfied. In the former case, $e P o A \geq 1.2$. In the latter case, $e P o A \geq \max \left\{\frac{x_{1}^{2}}{C_{\text {opt }}\left(x_{1}\right)}, \frac{\ell_{2}\left(r^{*}-x_{1}\right) r^{*}}{C_{\text {opt }}\left(r^{*}\right)}\right\}$. This completes the proof.

It remains to bound

$$
\min _{x_{1} \leq 1} \max \left\{\frac{x_{1}^{2}}{C_{\text {opt }}\left(x_{1}\right)}, \min _{r^{*} \geq 1} \frac{\ell_{2}\left(r^{*}-x_{1}\right) r^{*}}{C_{\text {opt }}\left(r^{*}\right)}\right\}=\min _{x_{1} \leq 1} \max \left\{\frac{x_{1}^{2}}{C_{\text {opt }}\left(x_{1}\right)}, \min _{r^{*} \geq 1} \frac{4 r^{*}(R+1)\left(r^{*}-x_{1}+R\right)}{R\left(4\left(r^{*}\right)^{2}+4 R r^{*}-R\right)}\right\}
$$

from below. We prove a lower bound of 1.191 . The term $x_{1}^{2} / C_{\text {opt }}\left(x_{1}\right)$ is increasing in $x_{1}$. Thus there is a unique value $\alpha_{1} \in[1 / 2,1]$ such that the first term is larger than 1.191 for $x_{1}>\alpha_{1}$. If the minimizing $x_{1}$ is larger than $\alpha_{1}$ we have established the bound. 
The second term is minimized for $r^{*}=\max \left\{1,\left(R+\sqrt{R^{2}+4 R^{2} x_{1}-4 R x_{1}^{2}}\right) /\left(4 x_{1}\right)\right\}$. Since $x_{1} \leq 1$ and hence $x_{1}^{2} \leq x_{1}$, we have $\left.\left(R+\sqrt{R^{2}+4 R^{2} x_{1}-4 R x_{1}^{2}}\right) /\left(4 x_{1}\right)\right) \geq 2 R / 4 \geq 1$ and hence

$$
r^{*}=\left(R+\sqrt{R^{2}+4 R^{2} x_{1}-4 R x_{1}^{2}}\right) /\left(4 x_{1}\right) .
$$

The second term is decreasing in $x_{1}$ and hence we may substitute $x_{1}$ by $\alpha_{1}$ for the purpose of establishing a lower bound. We now specialize $R$ to $21 / 10$. For this value of $R$ and $x_{1}=\alpha_{1}$

$$
\left.\frac{\ell_{2}\left(r^{*}-\alpha_{1}\right) r^{*}}{C_{o p t}\left(r^{*}\right)}\right|_{r^{*}=\left(R+\sqrt{R^{2}+4 R^{2} \alpha_{1}-4 R \alpha_{1}^{2}}\right) /\left(4 \alpha_{1}\right) \text { and } R=21 / 10} \geq 1.191 \text {. }
$$

This completes the proof of the lower bound.

We next argue that the construction of Section 4 is optimal. Equations (5) of Section 4 for $b_{1}=0$ and Equation (12) agree. Hence our refined solution is optimal.

\section{Open Problems}

Clearly the ultimate goal is to design coordination mechanisms that work for general networks. In the case of parallel links that we studied, we showed that our mechanism approaches $4 / 3$, as the number of links $k$ grows. It is still an open problem to show a bound of the form $4 / 5-\alpha$, for some strictly positive $\alpha$. A possible approach could be to use the ideas of Section 4 Another approach would be to define the benign case more restrictively. Assuming $R_{i}=8$ for all $i$, we would call the following latencies benign: $\ell_{1}(x)=x$, and $\ell_{i}(x)=1+\varepsilon \cdot i+x / 8^{i}$ for $i>1$ and small positive $\varepsilon$. However, Opt starts using the $k$-th link shortly after $1 / 2$ and hence uses an extremely efficient link for small rates.

Also, our results hold only for affine original latency functions. What can be said for the case of more general latencies, for instance polynomials? On the more technical side, it would be interesting to study whether our lower bound construction of Section 5 can be extended to modified latency functions $\hat{\ell}$ that do not need to satisfy monotonicity.

Acknowledgements We would like to thank Elias Koutsoupias, Spyros Angelopoulos and Nicolás Stier Moses for many fruitful discussions.

\section{References}

[1] E. Angel, E. Bampis, and F. Pascual. Truthful algorithms for scheduling selfish tasks on parallel machines. Theor. Comput. Sci., 369(1-3):157-168, 2006.

[2] E. Angel, E. Bampis, F. Pascual, and A.-A. Tchetgnia. On truthfulness and approximation for scheduling selfish tasks. J. Scheduling, 12(5):437-445, 2009.

[3] Y. Azar, K. Jain, and V. S. Mirrokni. (Almost) optimal coordination mechanisms for unrelated machine scheduling. In SODA, pages 323-332,2008.

[4] D. Bernstein and T. E. Smith. Equilibria for networks with lower semicontinuous costs: With an application to congestion pricing. Transportation Science, 28(3):221-235, 1994.

[5] V. Bonifaci, M. Salek, and G. Schäfer. On the efficiency of restricted tolls in network routing games. In SAGT, pages 302-313, 2011. 
[6] I. Caragiannis. Efficient coordination mechanisms for unrelated machine scheduling. In SODA, pages 815-824, 2009.

[7] G. Christodoulou, L. Gourvès, and F. Pascual. Scheduling selfish tasks: about the performance of truthful algorithms. In COCOON, pages 187-197, 2007.

[8] G. Christodoulou, K. Mehlhorn, and E. Pyrga. Improving the Price of Anarchy for Selfish Routing via Coordination Mechanisms. In ESA, pages 119-130, 2011.

[9] G. Christodoulou, E. Koutsoupias, and A. Nanavati. Coordination mechanisms. Theor. Comput. Sci., 410(36):3327-3336, 2009.

[10] R. Cole, J. R. Correa, V. Gkatzelis, V. Mirrokni, and N. Olver. Inner product spaces for minsum coordination mechanisms. In STOC, 2011.

[11] R. Cole, Y. Dodis, and T. Roughgarden. Pricing network edges for heterogeneous selfish users. In STOC, pages 521-530, 2003.

[12] R. Cole, Y. Dodis, and T. Roughgarden. How much can taxes help selfish routing? J. Comput. Syst. Sci., 72(3):444-467, 2006.

[13] J. R. Correa, A. S. Schulz, and N. E. Stier-Moses. A geometric approach to the Price of Anarchy in nonatomic congestion games. Games and Economic Behavior, 64:457-469, 2008.

[14] S. C. Dafermos and F. T. Sparrow. The traffic assignment problem for a general network. Journal of Research of the National Bureau of Standards, Series B, 73B(2):91-118, 1969.

[15] S. Dafermos. An extended traffic assignment model with applications to two-way traffic. Transportation Science, 5:366-389, 1971.

[16] A. de Palma and Y. Nesterov. Optimization formulations and static equilibrium in congested transportation networks. In CORE Discussion Paper 9861, Université Catholique de Louvain, Louvainla-Neuve, pages 12-17, 1998.

[17] L. Fleischer. Linear tolls suffice: New bounds and algorithms for tolls in single source networks. Theor. Comput. Sci., 348(2-3):217-225, 2005.

[18] L. Fleischer, K. Jain, and M. Mahdian. Tolls for heterogeneous selfish users in multicommodity networks and generalized congestion games. In FOCS, pages 277-285, 2004.

[19] N. Immorlica, L. Li, V. S. Mirrokni, and A. Schulz. Coordination mechanisms for selfish scheduling. In WINE, pages 55-69, 2005.

[20] G. Karakostas and S. G. Kolliopoulos. Edge pricing of multicommodity networks for heterogeneous selfish users. In FOCS, pages 268-276, 2004.

[21] G. Karakostas and S. G. Kolliopoulos. The efficiency of optimal taxes. In CAAN, pages 3-12, 2004.

[22] K. Kollias. Non-preemptive coordination mechanisms for identical machine scheduling games. In SIROCCO, pages 197-208, 2008.

[23] E. Koutsoupias and C. Papadimitriou. Worst-case equilibria. Computer Science Review, 3(2):6569, 2009. 
[24] P. Marcotte and M. Patriksson. Traffic equilibrium. In Transportation, volume 14 of Handbooks in Operations Research and Management Science, chapter 10, pages 623-713. North-Holland, 2007.

[25] N. Nisan, T. Roughgarden, E. Tardos, and V.V. Vazirani. Algorithmic Game Theory. Cambridge University Press, 2007.

[26] M. Patriksson. The Traffic Assignment Problem: Models and Methods. V.S.P. Intl Science, 1994.

[27] T. Roughgarden. Designing networks for selfish users is hard. In FOCS, 2001.

[28] T. Roughgarden and É. Tardos. How bad is selfish routing? J.ACM, 49:236-259, 2002.

[29] J. G. Wardrop. Some theoretical aspects of road traffic research. In Proceedings of the Institute of Civil Engineers, Part II, volume 1, pages 325-378, 1952. 